\title{
Systemic lupus erythematosus. II. Observations on the occurrence of exacerbations in the disease course: Dutch experience with 110 patients studied prospectively
}

\author{
A J G SWAAK, ${ }^{1}$ J C NOSSENT, ${ }^{1}$ W BRONSVELD ${ }^{3}$ A VAN ROOYEN ${ }^{2}$ \\ E J NIEUWENHUYS, ${ }^{2}$ L THEUNS, ${ }^{1}$ AND R J T SMEENK ${ }^{2}$
}

From the ${ }^{1}$ Department of Rheumatology, Daniel den Hoed Clinic, Rotterdam; the ${ }^{2}$ Department of Auto-Immune Diseases, Central Laboratory for Blood Transfusion, Amsterdam; and the ${ }^{3}$ Department of Internal Medicine, Medical Center, Alkmaar

SUMMARY The incidence of exacerbations in the disease course was investigated in 110 patients with systemic lupus erythematosus (SLE) who were studied prospectively at our institute for lupus research. At the time of disease onset and diagnosis the male patients were much older than the female patients (about 10 years); exacerbation frequency during follow up was increased in the male patients. The follow up data showed that if a patient with SLE was prone to develop an exacerbation this mostly took place within the first five years of follow up. It could be calculated that after fulfilling the American Rheumatism Association criteria only $56 \%(62 / 110)$ of the patients developed a subsequent exacerbation. Features at the time of diagnosis, distinguishing those patients who developed a subsequent exacerbation from those who did not, were haemolytic anaemia, the presence of anti-Sm antibodies, and a falsely positive serological test for syphylis. At the time of diagnosis, however, the prevalences of these features were low; for haemolytic anaemia, anti-Sm antibodies, and a falsely positive serological test for syphilis they amounted to $40 \%, 5 \%$, and $12 \%$ respectively.

Nowadays patients with systemic lupus erythematosus (SLE) have a much better prognosis than 20 years ago. ${ }^{1-3}$ This may be due either to improved treatment or to the fact that in recent years less severely ill patients have also been recognised. This increased recognition is clearly promoted by the widely employed use of the revised American Rheumatism Association (ARA) criteria for the diagnosis of SLE. ${ }^{4}$ It is relatively easy to establish the diagnosis of SLE if a patient shows all the characteristic manifestations of the disease, including fever, arthritis, cutaneous lesions, sterile effusion in serous cavities, and nephritis. It remains difficult, however, to establish the diagnosis when

Accepted for publication 30 September 1988

Correspondence to Dr A J G Swaak, Department of Rheumatology, Daniel den Hoed Clinic, Groene Hilledijk 301, 3075 EA Rotterdam, The Netherlands. the characteristic skin and joint manifestations, which mostly form the initial complaints of the patient, are not present.

Many reports have focused on questions related to treatment or prognosis of patients who had developed renal, cardiopulmonary, and other organ manifestations of the disease. ${ }^{5-7}$ Incidence frequencies of the various disease features have always been described retrospectively or by transversal studies, or both, of a given number of patients with SLE. It must be emphasised that the disease course is characterized by fluctuations in activity. Disease activity may be profound at some times but may be followed by periods of relative absence of symptoms. It is also important to realise that SLE may have long periods of remission; some patients may live for 10 to 15 years, completely free from active symptoms. ${ }^{8-11}$

The studies we started in 1970 (see preceding 
paper and refs 12-14) now permit a prospective evaluation of the disease course of a large group of patients. The aim of the present study was to evaluate which patients with SLE will develop an exacerbation and with what frequency, and whether factors can be identified to predict the development of an exacerbation. Furthermore, the influence of factors, like sex, age of onset, age of diagnosis, and clinical signs at onset, on the disease course have been studied.

\section{Patients and methods}

\section{PAT I E N T S}

All patients fulfilling the preliminary ARA criteria for SLE ${ }^{15}$ and who were seen and followed up by either AJGS or WB at our lupus outpatient clinics since 1970 , took part in the study. The patients were first seen at our department for diagnostic reasons. At that time the diagnosis SLE was either confirmed or rejected. If patients later developed SLE they were then also incorporated in this study. One hundred and ten patients were selected for evaluation; they have been followed up until now at our outpatient clinic for lupus research. Demographic features of these 110 patients have been given in the preceding paper.

\section{DISEASE ACTIVITY}

Disease activity can be divided into minor and major symptoms, ${ }^{12} 1316$ which have been defined in the preceding paper. Episodes in which disease features could be explained by causes other than SLE were excluded. Patients with pre-existing renal involvement were considered to be inactive when no significant alterations took place in the creatinine values, proteinuria, and urine sediment.

D I A G N OS IS

When our studies started in 1970 the ARA prelimin- ary criteria ${ }^{15}$ were used for the diagnosis of SLE? We continued to use these criteria until the end $\vec{f}$ the study. Use of the revised criteria, ${ }^{4}$ however would not have significantly changed the data of disease onset or diagnosis.

Time of diagnosis was defined as the moment which the patient fulfilled four or more of the AR\& criteria. Onset of disease was defined as the date which the patient had complaints related to the disease for the first time.

For the calculation of exacerbation frequency anct incidence the moment of diagnosis was not colas sidered to be an exacerbation, even if the patient dia show major disease features at that moment. Wherbo after a minimum period of three months, a recupo rence or an exacerbation took place this was considered to be the first exacerbation. These definitions are, admittedly, quite arbitrary, but we feel that one can only speak of an exacerbation som $\vec{e}$ period after the diagnosis SLE has been establishec:

All clinical data were stored in a Wang persona computer, using the Lotus $1-2-3$ program. Clinicad signs at the onset of the disease, time of diagnos: (fulfilling the ARA criteria), time interval between onset and diagnosis, age and sex, number and time of exacerbation (if relevant) were all recordec. Statistical evaluation of results was undertaken witi analysis of variance for mean values, with Student two tailed $t$ test for group differences. $\mathrm{p}$ Values $<0.05$ were considered significant.

\section{Results}

DIFFERENCES BETWEEN PATIENTS WITH SL DEVELOPING OR NOT DEVELOPING

AN EXACERBATION

As described in the preceding paper more male thain female patients with SLE died during our follow uP study. Also, 12 of the 16 men (75\%) developed ab exacerbation, in contrast with 50 of the 94 femae

Table 1 Comparison of those patients with systemic lupus erythematosus who developed one or more exacerbationg (group A) and those who still had not developed an exacerbation at the end of the study (group B)

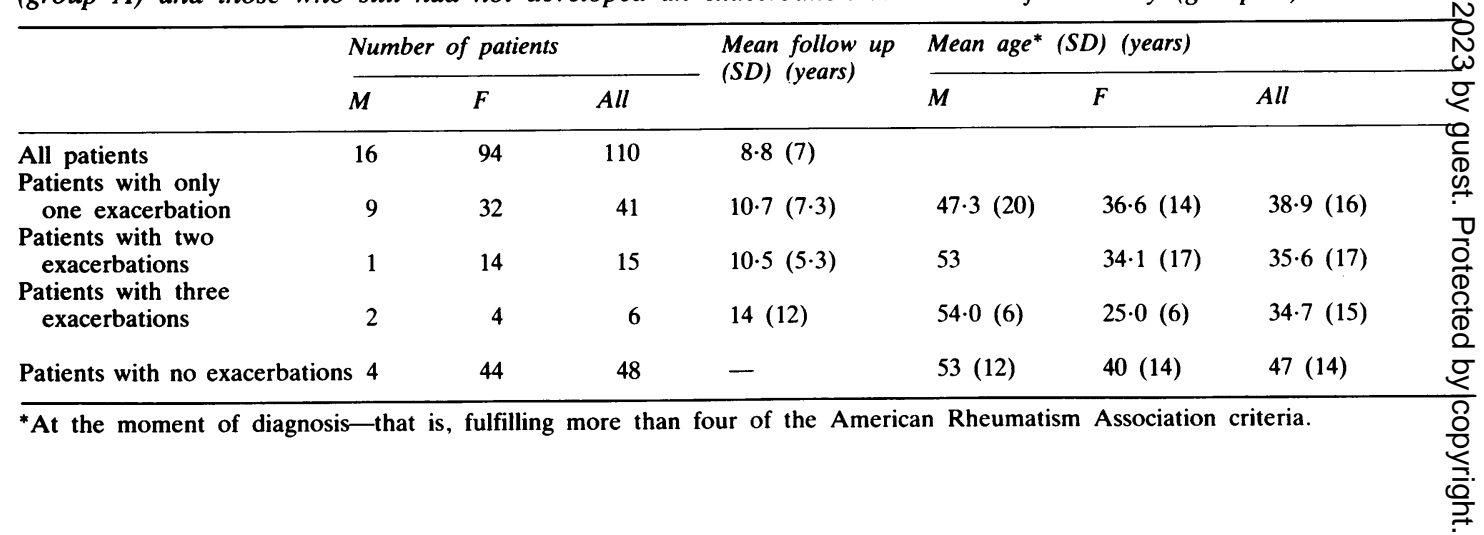


patients $(53 \%)$. The exacerbation frequency of men and women who died was comparable (1.42 and 1.86 respectively). Overall, at the onset of disease and at the time of diagnosis the male patients were about 10 years older than the female patients. Table 1 indicates the differences between those patients with SLE who developed an exacerbation (group A) and those who still had not developed an exacerbation at the end of the study (group B).

No significant differences in the mean follow up duration were found between patients of groups A and B. Only those patients who developed three exacerbations had been studied somewhat longer. A remarkable difference was found between the mean age of the patients of both groups at the moment of diagnosis: patients of the first group were clearly younger than those of the second group $(p=0.005)$. There was also an indication (not significant) that the younger patients were more prone to develop an exacerbation. These facts are further illustrated by the mean age of the patients at the moment of their exacerbation (Table 2). No differences in mean time intervals between the moment of diagnosis and the first exacerbation, or between the first and the second exacerbation, existed between those patients who developed one or two exacerbations (Table 3 ).

Figure 1 shows the time intervals between the moment of diagnosis and an exacerbation for all patients developing one or more exacerbations. Figure 2 summarises cumulatively the results of Table 3 and Fig. 1.

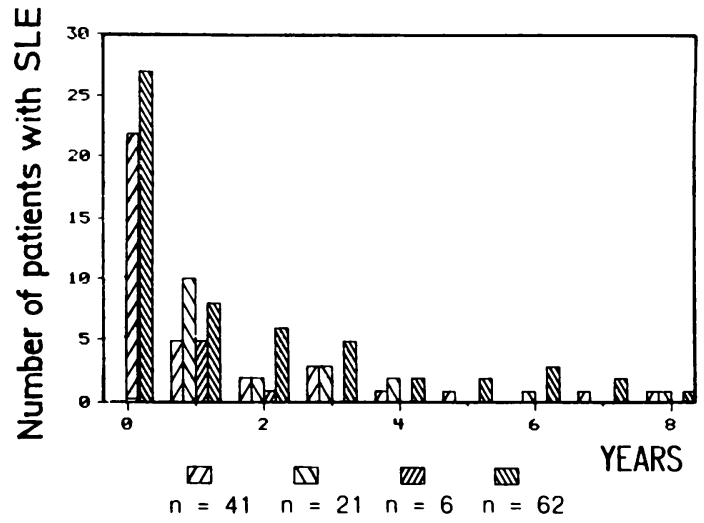

Fig. 1 Time intervals (years) between the occurrence of an exacerbation and the diagnosis. $\mathbb{N}=$ all patients with one or more exacerbation ( $n=62) ; \quad=$ patients with only one exacerbation $(n=41) ; \mathbb{N}=$ patients developing a second exacerbation $(n=21)$. The time intervals are shown between the time of diagnosis and the second exacerbation; $\mathbb{Z}=$ patients with a third exacerbation $(n=6)$. The time intervals are shown between the time of diagnosis and the third exacerbation.

From these data it is clear that if a patient with SLE is prone to develop an exacerbation this will take place within the first five years of follow up. The first exacerbations mostly took place three

Table 2 Mean age of the patients at the moment of their exacerbation

\begin{tabular}{|c|c|c|c|c|c|c|}
\hline & \multicolumn{3}{|c|}{ Number of patients } & \multicolumn{3}{|c|}{$\begin{array}{l}\text { Mean ages }(S D) \text { at the } \\
\text { indicated exacerbations (years) }\end{array}$} \\
\hline & $M$ & $F$ & All & $M$ & $F$ & All \\
\hline First exacerbation & 9 & 32 & 41 & $47(18)$ & $39(15)$ & $41(16)$ \\
\hline Second exacerbation & 1 & 14 & 15 & 55 & $38(17)$ & 40 (17) \\
\hline Third exacerbation & 2 & 4 & 6 & - & - & $36(14)$ \\
\hline
\end{tabular}

Table 3 Mean time intervals between time of diagnosis and the first exacerbation, and between first and second exacerbations

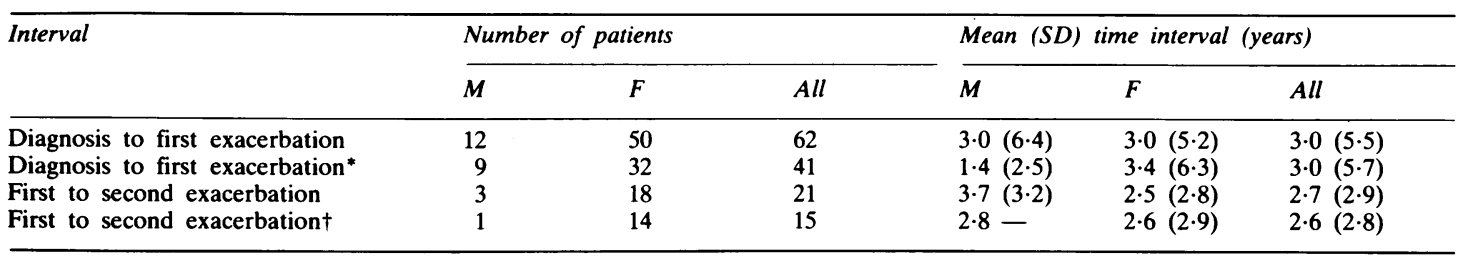

* Patients developing a second or third exacerbation were excluded.

†Patients developing a third exacerbation were excluded. 
458 Swaak, Nossent, Bronsveld, van Rooyen, Nieuwenhuys, Theuns, Smeenk

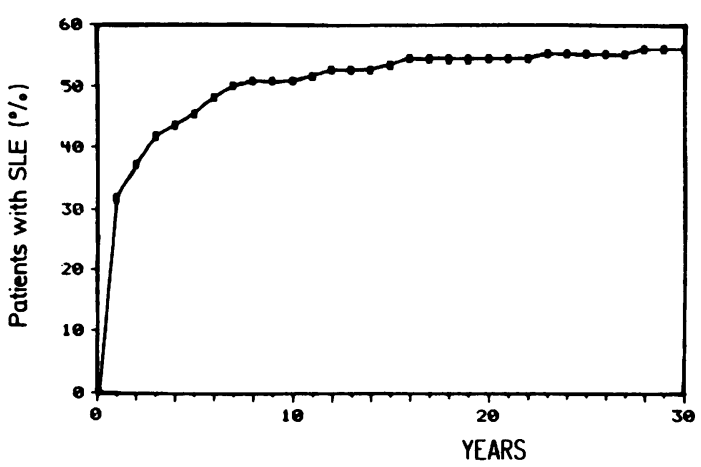

Fig. 2 Cumulative percentage of patients $(n=110)$ who developed an exacerbation as a function of the time passed since the patients fulfilled the diagnostic criteria for systemic lupus erythematosus.

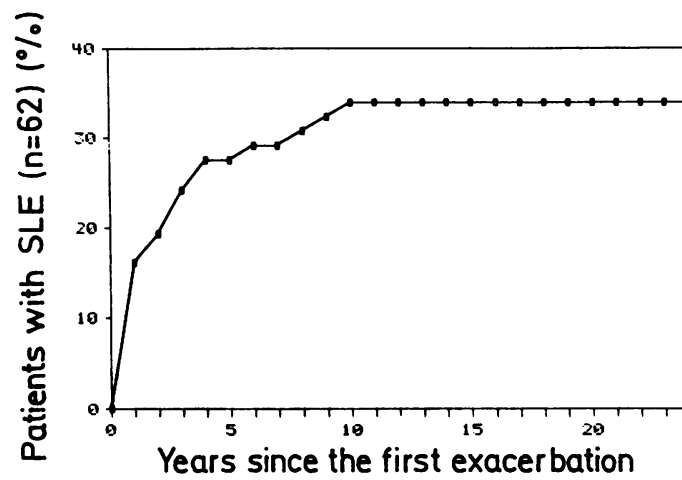

Fig. 3 Cumulative percentage of patients $(n=62)$ who developed a second exacerbation as a function of the time passed since the first exacerbation.

Table 4 Comparison of the clinical signs* at the time of diagnosis (more than four American Rheumatism Association criteria) in those patients who never showed evidence of an exacerbation (group B) and in those who developed one or mors exacerbation (group A) during the follow up

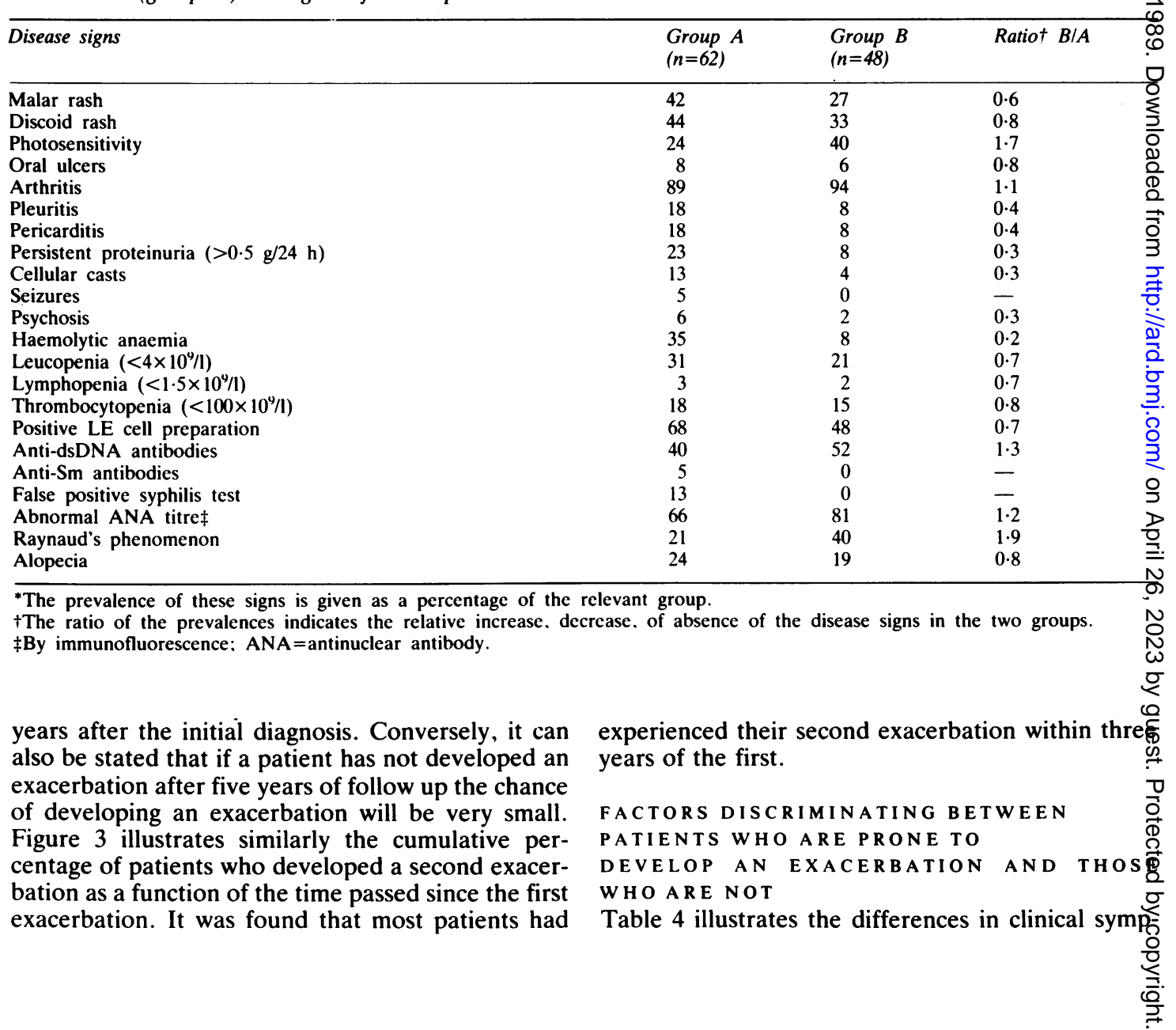


toms at the time of diagnosis between both groups of patients. The relative increase or absence of a given clinical symptom is calculated as a ratio (quotient of the prevalences).

The significant differences were the increased prevalence in group B patients of haemolytic anaemia, anti-Sm antibodies, and the falsely positive serological test for syphylis.

\section{Discussion}

Once a patient has been diagnosed as having SLE there may be a tendency to attribute any subsequent illness to this disease. Symptoms of SLE may be caused by a variety of other diseases not directly related to the underlying SLE, however. ${ }^{17}$ It is therefore important to analyse profoundly any new clinical development occurring during the disease, paying special attention to the possibility that the symptoms may or may not be related to the SLE.

A number of reports have given incidences of various disease symptoms in a given group of patients. ${ }^{18-20}$ In this paper we examined how many times an exacerbation took place in the disease course of SLE and attempted to determine whether any factors distinguished those patients who developed a subsequent exacerbation from those who did not. When the results are considered it should be borne in mind that SLE is a chronic disease, which may show varying periods of activity. Even if it runs an unremitting course, wide differences in rates of progression can be seen. Concurrently the patient gets older and may suffer other diseases and experiences causing physiological changes. All these factors may influence this evaluation. Therefore, we have focused on acute illness only (according to the definitions of a clinical exacerbation). In this way a progressive but slow decrease in renal function, taking place during the 10 years or more of follow up of a given patient, will be neglected.

In the current study most patients could be studied from the moment the diagnosis was made; still, time periods between disease onset and diagnosis varied. Although a lot of drawbacks have been overcome by the prospective nature of this study, the variable length of the follow up for each patient may influence our data. Several factors characterised patients who were prone to develop an exacerbation. Firstly, a relatively increased exacerbation incidence was found in male patients compared with female patients. Secondly, at the time of diagnosis the presence of haemolytic anaemia, anti-Sm antibodies, and a falsely positive serological test for syphilis were found at a higher incidence in patients developing one or more exacerbations.

This study shows two other important things that may have prognostic clinical significance. Firstly, only half of the patients will develop an exacerbation after the initial flare up which led to the diagnosis SLE. Secondly, if a patient with SLE has shown no evidence of an exacerbation during five years of follow up then the chance of the patient developing an exacerbation in the future will be very small.

These results illustrate that after the diagnosis SLE has been confirmed in a patient further organ involvement will not always take place; it occurs in approximately $50 \%$ of the patients with SLE.

We wish to thank Thea van Vlijmen, Mariska Drinkwaard, and M Pillay for their assistance in preparing the manuscript. This study was financially supported by the Netherlands League against Rheumatism.

\section{References}

1 Estes D, Christian C L. The natural history of systemic lupus erythematosus by prospective analysis. Medicine (Baltimore) 1971; 50: 85-95.

2 Merrell M. Schulman L E. Determination of prognosis in chronic disease, illustrated by systemic lupus erythematosus. $J$ Chronic Dis 1955: 1: 12-32.

3 Urman J D, Rothfield N F. Corticosteroid treatment in systemic lupus erythematosus: survival studies. JAMA 1977; 238: 2272-6.

4 Tan E M, Cohen A S, Fries J F, et al. The 1982 revised criteria for classification of systemic lupus erythematosus. Arthritis Rheum 1982; 25: 1271-7.

5 Wallace D J, Podell T E, Weiner J M, et al. Lupus nephritis: experience with 230 patients in a private practice from 1950 to 1980. Am J Med 1982; 72: 209-20.

6 Wallace D J. Podell T E, Weiner J, Klinenberg J R, Forouzesh S, Dubois E L. Systemic lupus erythematosus-survival patterns: experience with 609 patients. JAMA 1981; 245: 934-8.

7 Urowitz M B, Bookman A A M, Kochler B E, Gordon D A, Smythe H A, Ogryzlo M A. The bimodal mortality pattern of systemic lupus erythematosus. Am J Med 1976; 60: 221-5.

8 Dubois E L. Systemic lupus erythematosus: recent advances in its diagnosis and treatment. Ann Intern Med 1956; 45: 163-84.

9 Ropes M W. Observations on the natural course of disseminated lupus erythematosus. Medicine (Baltimore) 1964; 43: $387-91$.

10 Ben-Asher S. Recurrent acute lupus erythematosus disseminatus: report of case which survived 23 years after onset of systemic manifestations. Ann Intern Med 1951; 34: 243-8.

11 Short C L. Bauer W. Reynold W E. Rheumatoid arthritis. A definition of the disease and a clinical description based on a numerical study of 293 patients and controls. Cambridge, Mass. Harvard University Press, 1957.

12 Swaak A J G. Groenwold J, Aarden L A. Feltkamp T E W. Detection of anti-dsDNA as diagnostic tool. Ann Rheum Dis 1981: 40: 45-9.

13 Swakk A J G, Groenwold J, Aarden L A. Statius van Eps L W, Feltkamp T E W. Prognostic valuc of anti-dsDNA in SLE. Ann Rheum Dis 1982; 41: 388-95.

14 Swaak A J G. Groenwold J. Bronsveld W. Predictive value of complement profiles and anti-dsDNA in systemic lupus erythematosus. Ann Rheum Dis 1986; 45: 359-66.

15 Cohen A S. Reynolds W F. Franklin E G, et al. Preliminary 
460 Swaak, Nossent, Bronsveld, van Rooyen, Nieuwenhuys, Theuns, Smeenk

criteria for the classification of systemic lupus erythematosus. Bull Rheum Dis 1971; 21: 643-8.

16 Lightfoot R W Jr, Hughes G R V. Significance of persisting serologic abnormalities in systemic lupus erythematosus. Arthritis Rheum 1976; 19: 837-43.

17 Stahl N I, Klippel J H, Decker J L. Fever in systemic lupus erythematosus. Am J Med 1979; 67: 935-40.

18 Dubois E L, ed. Lupus erythematosus. A review of the current status of discoid and systemic lupus erythematosus and their variants. 2nd ed. Los Angeles: University of Southern California Press, 1974.

19 Bulgrin J G, Dubois E L, Jacobsen G. Chest roentgenographic changes in systemic lupus erythematosus. Radiology 1960; 74: 42-9.

20 Larson D L. Systemic lupus erythematosus. Boston: Little, Brown, 1961. 